\section{MedienPädagogik}

www. medienpaed.com
Zeitschrift für

Theorie und Praxis

der Medienbildung

ISSN 1424-3636

Themenheft Nr. 21: Partizipationschancen im Kulturraum Internet nutzen und gestalten - Das Beispiel Web 2.0

\title{
Kakophonie und Selbstorganisation in der digitialen Agora
}

Hans Geser

\begin{abstract}
Die - in jüngster Zeit immer deutlicher hervortretende - Doppelfunktion des Internets besteht darin, die politische Öffentlichkeit einerseits aus der traditionellen Dominanz «vermachteter» monologischer Abwärtskommunikation zu befreien und andererseits eine polyvalente Plattform zur Verfügung zu stellen, auf der alle Formen technisch gestützter Kommunikation ohne Medienbrüche prozessiert und ineinander übergeführt werden können. Dank zeitunabhängiger Verfügbarkeit aller Inhalte profiliert sich die Internet-Öffentlichkeit zudem durch eine historische Tiefendimension, die mit der kurzfristigen Aktualitätsorientierung der konventionellen Medien scharf kontrastiert.

Der immensen Ausweitung niederschwelliger öffentlicher Partizipationschancen und (spontan-individuell bestimmter) interaktiver Horizontalkommunikationen stehen allerdings viel geringere Gewinne an kollektiver Organisations- und Aktionskapazität gegenüber, weil digitale politische Engagements vergleichsweise unverbindlich bleiben und weil im Stadium der Kampagne meist keine formalisierten Strukturen entstehen, wie sie für die nachfolgenden Stadien aktiver Konfliktaustragung und Politikrealisierung notwendig sind. Da traditionelle Eliten kaum an Onlinekommunikation teilnehmen, ist bisher keine integrierte Gesamtöffentlichkeit entstanden, die die Vorteile massenmedialer Diffusion und digitaler Kommunikation in sich vereinigt.
\end{abstract}

\section{Einleitung}

Lange vor den jüngeren Konzentrations- und Monopolisierungstendenzen im Pressewesen hat Jürgen Habermas bereits 1962 auf die demokratiefeindlichen Wirkungen einer von monologischen Massenmedien dominierten «vermachteten Öffentlichkeit» hingewiesen, die der die seit der Aufklärung geforderten «herrschaftsfreie Kommunikation mündiger Bürger keinen Raum gibt, weil politische Macht und Kapitalbesitz über den Zugang zu den Sendekanälen entscheiden. Trotz seiner technologischen Modernität kontrastiert dieses System der monologischen Einwegmedien zunehmend mit fast allen übrigen Institutionen, in denen sich seit den späten 1960er eine Verschiebung zu partizipativeren und dialogischeren Kommunikationsformen vollzogen hat. So ist in den Schulen der traditionelle 
Frontalunterricht dem offeneren Gruppen- und Teamunterricht gewichen, in Unternehmungen werden Mitarbeiter im Rahmen der «lean production» stärker in die betriebliche Verantwortung integriert und in den Parteien ist der die Tradition des Grossvaters fortführende Stammwähler dem unberechenbaren Wechselwähler gewichen, der sich seine eigene unabhängige Meinung bildet und in jeder neuen Sachfrage neu überzeugt werden will.

Vor allem sind seit den 1970er-Jahren «Neue Soziale Bewegungen» entstanden, die sich nicht mehr auf charismatische Führerschaft und monopolistisch verwaltete Ideologien abstützen, sondern in ihrem Kern aus relativ offenen dezentralen Netzwerken semi-autonomer Gruppierungen und Aktivisten bestehen, die andauernde Diskurse über die Priorität verschiedener Ziele und Aktivitäten führen.

Es ist bemerkenswert, dass sich bereits seit den späten 60er Jahren des letzten Jahrhunderts trotz dieser restriktiven Medienöffentlichkeit auf der Basis von Flugblättern, FAX-Botschaften, Fotokopien und billigen Taschenbüchern eine vielfältige Szene von marginalen «weak publics» ausgebildet hat, in der Feministinnen, Homosexuelle, Obdachlose, Migrantengruppen, religiöse Fundamentalisten usw. ihre eigenen, voneinander oft astronomisch weit entfernten Diskurse führen (vgl. z. B. Fraser 1992, $118 \mathrm{ff}$.$) .$

All diese wachsenden Bedürfnisse medialer Selbstartikulation habe ganz objektiv das Bedürfnis nach ganz neuartigen Medientechnologien anwachsen lassen, die einen niederschwelligeren Zugang zu öffentlicher Kommunikation gewähren und die neben traditionellen "one-to many» Emissionen auch vertikale Aufwärtskommunikation («many to one») und sowie den horizontalen Austausch («many to many») technisch unterstützen.

So richten sich heute berechtigte Hoffnungen auf die neuen weltweiten Computernetze, deren Hauptfunktion ja darin besteht, ihren Nutzern unabhängig von Ort, Zeit und sozialen Kontrollen äusserst niederschwellige Möglichkeiten zur Information und Kommunikation in der globalen Öffentlichkeit zugänglich zu machen. Wurde das Web 1.0 der späten 90er Jahre noch primär als ein alternativer Kanal für monologische Emissionen begriffen, sind im neueren Web 2.0 die für das Internet spezifischen Aspekte dezentralisiert-interaktiver Kommunikation stärker in den Vordergrund getreten. Wider Erwarten haben sich die durchschnittlichen User keineswegs als rein konsumptive "Couch Potatoes» erwiesen, sondern als «Prosumenten», die ihre kommunikativen Aktivitäten zunehmend im öffentlichen Raum (vor allem auf «Social Network Sites» wie Facebook oder in Chats, Blogs, Diskussionsformen und Wikis) stattfinden lassen. ${ }^{1}$

1 So hat eine Nielsen-Studie vom Juni 2010 gezeigt, dass amerikanische Nutzer durchschnittlich 36\% ihrer Onlinezeit mit interaktiven «Social Media» verbringen, wobei sich der Schwerpunkt immer mehr von bilateralen Emails auf multilaterale «Social Network Sites» (Facebook, Twitter u. a.) verschiebt (Nielsen Wire 2010). 
Aus pädagogischer Sicht ist bedeutsam, dass hier ohne institutionelle Planung und Steuerung millionenfache spontane Selbstqualifizierungsprozesse stattfinden, in denen gelernt wird, sich mit Wort, Ton, Bild und Video öffentlich zu artikulieren und auf die Artikulationen anderer zu reagieren.

Im folgenden sollen einige Rahmenbedingungen und Basismerkmale der - momentan erst umrisshaft erkennbaren «neuen politischen Öffentlichkeit» diskutiert werden, wie sie aus dem Zusammenwirken technischer Potentialitäten mit soziokulturellen und psychologischen Gestaltungskräften (bzw. Blockierungen) allmählich entsteht.

\section{Breitere Chancen kollektiver politischer Gruppenbildung und Artikulation}

Die Gewinne für politische Kommunikation sind bisher paradoxerweise vor allem in jenen peripheren Regionen deutlich geworden, wo es an konventionellen offline-Kanälen mangelt: z. B. wenn es den Zapatisten gelingt, sich aus dem tiefsten südmexikanischen Urwald zentral in die Weltöffentlichkeit einzuklinken; wenn die «Arab Association of Gays and Lesbians»² diesen einzigen Weg nutzt, um ihre Minderheitsrechte gegen staatliche Repression zu artikulieren; oder wenn emigrierte Sudanesen - wie weiland Heine und Börne in Paris -im Netz eine "Offshore-Öffentlichkeit» zum Leben erwecken, in der man frei über die künftigen Entwicklungen ihres autoritär gelenkten Heimatstaates diskutiert.

Je grösser und je heterogener die Netzgemeinde, desto besser die Chance, auch für höchst abseitige Themen und Meinungen weltweit auf eine für die Gründung einer Gruppe hinreichende Zahl Gleichgesinnter zu stossen. Bei manchen von ihnen mag es sich um den embryonalen Keim einer neuen sozialen Bewegungsgruppe handeln, die in Zukunft vielleicht expansiv an die Öffentlichkeit tritt und Zugang zu konventionelleren Medienkanälen gewinnt. Während die Einwirkung auf das politische «Agenda Setting» früher gut organisierten Parteien und Verbänden vorbehalten war, So bietet das Internet heute niederschwellige Möglichkeiten, um durch Austesten von Konsenschancen neue Themen in die politische Diskussion zu bringen oder gar eine neue «Soziale Bewegung» zu initiieren. So hat Franziska Heine (eine 27-jährige Webdesignerin) mit 134000 Unterzeichneten mühelos die bisher grösste deutsche Onlinepetition (gegen Internetsperren) realisiert, und Susanne Wiest (eine Tagesmutter aus Greifswald) hat die Diskussion über ein bedingungslosen Grundeinkommen belebt, indem sie für ihre Forderung in kurzer Zeit 53000 «Mitstreiter» fand.

So manifestieren sich neue politische Strömungen und Parteibildungen heute in viel früherem Stadium be-reits in öffentlich klar sichtbarer Form: mit der Folge, dass politischen Themen und die daran beteiligten Gruppen unvorhersehbarer variieren, und dass generell die Konsenschancen schwinden, weil sich die bis-

2 http://www.glas.org/ 
herige «schweigende Mehrheit» zunehmend in eine volatile Vielfalt von «sprechenden Minderheiten» transformiert. Aus demselben Grund kann die Netzkommunikation den inneren Zusammenhalt konsensbedürftiger Organisationen (Parteien, Verbände, religiöse Sekten u. a.) bedrohen, weil deren Führungsorgane kein Monopol mehr über den Zugang zur Öffentlichkeit mehr besitzen, da jede dissidente Minderheit ohne Kosten und Mühe in der Lage ist, ihre abweichenden Positionen eigenständig in der Netzöffentlichkeit zu publizieren (Geser 2001). Für die Führungseliten wird es dadurch schwieriger, das Wort «wir» zu verwenden - und damit einen kollektiven Konsens zu unterstellen. Denn wenn alle sich mühelos zu Wort melden können, wird es viel wahrscheinlicher, dass faktisch bestehender Dissens auch offensichtlich wird und bisher für unproblematisch gehaltene Konsensannahmen sich als unhaltbar erweisen (Geser 1996).

Polyvalente Einheitsplattform für vertikale und horizontale Kommunikationen Die Computernetze ermöglichen es, sämtliche Formen technisch vermittelter Kommunikation, die bisher auf völlig getrennten Kanälen gesendet worden sind, auf derselben Plattform stattfinden zu lassen und in einem umfassenden Gesamtzusammenhang zu integrieren (Oblak 2003). Keine Fotokopien sind mehr nötig, um eine schriftliche Einsendung einem grösseren Kreis zugänglich zu machen; digitale Sitzungsprotokolle haben bereits die Form, die sie für ihre Veröffentlichung brauchen, und formelle Dokumente können sehr leicht in den privaten Emailverkehr oder in öffentliche Forumsdiskussionen eingeschlossen werden.So impliziert die Teilnahme an einer typischen Online-Diskussion (z. B. bei Telepolis oder in der «Welt»), dass ich im selben Medium, in dem ich den redaktionellen Artikel lese,

- meine eigene Reaktion darauf publizierere

- die Stellungnahme anderer Leser zur Kenntnis nehme

- meine Meinung zu diesen anderen Stellungnahmen (z. B. dichotom als «Zustimmung» vs. «Ablehnung») deponiere;

- zur Kenntnis nehme, wie andere meinen eigenen Beitrag (sowie alle übrigen Beiträge) werten.

Bereits dieses sehr bescheidene Instrumentarium von Web 2.0 Technologien eröffnet eine Verdichtung und Dynamisierung der öffentlichen Meinungsbildung, die in früheren Online-Diskussionsformen noch undenkbar war. Denn indem die aus allen vergangenen Voten entstandene aktuellen Meinungskonstellatioen in Realzeit offen zu tage liegen, sehen sich alle Teilnehmer dazu fähig, ihre eigenen Positionen im Lichte dieser aggregierten öffentlichen Meinungsbildung zu verorten und evtl. reflexiv neu zu positionieren. 
Innerhalb von wenigen Minuten kann sich so z. B. auf dem Wege rein horizontaler Kommunikation eine Konsensbildung vollziehen, die früher nur durch vertikale Persuasion seitens professioneller Medienkommunikatoren möglich war.

Allerdings stellt man aber fest, dass die traditionellen Medienschaffenden ebenso wie die politischen Eliten bisher wenig Neigung zeigen, die durch sie ausgelösten Reaktionen auch nur explizit zur Kenntnis zu nehmen, geschweige denn materiell darauf zu reagieren. ${ }^{3}$ Tatsächlich haben traditionelle "Offline Eliten» im Netz viel zu verlieren, weil sie in einem Medium, das alle User zu gleichrangigen Lieferanten von Textvoten degradiert, keine Möglichkeiten sehen, ihre angestammte Autoritätsposition (die in offline Veranstalungen z. B. durch einen bevorzugten Sitz im Frontpanel zum Ausdruck kommt) zu bewahren. Überdies belasten sie sich nur selbst mit Anfragen, deren Beantwortung wieder wertvolle Zeit verschlingen kann - ohne dass gesichert ist, dass eine über einzelne Rezipienten weit hinausreichende Wirkung erzielt werden kann. So kommt es, dass die von den Führungseliten allein gelassenen (oder im besten Fall aktiv zensurierten) Netizens häufig irrelevanten «loser created content» produzieren, der die realen politischen Deliberationsund Entscheidungsprozesse in keiner Weise bestimmt. ${ }^{4}$

\section{Gesteigerte Individualisierung als Hindernis politischer Konsensbildung und Organisation}

Wenn sich politische Aktivität als Partizipation an Sitzungen, Versammlungen oder andern Anlässen der face-to-face-Kommunikation vollzieht, begeben sich die Individuen in ein dichtes soziales Feld, innerhalb dem sich mannigfachen Beeinflussungen und Kontrollen unterliegen (Geser 2002). Beispielsweise werden sie häufig genötigt, sich auch die Voten unliebsamer Redner anzuhören, bis zum Ende einer Zusammenkunft auszuharren, einen von niemand anderem kritisierten Beschluss selbst bei abweichender Meinung stillschweigend mitzutragen oder an Demonstrationen in den Chor der Protestierenden einzustimmen. Dies alles hat zur Folge, dass Intensität und Form des partizipativen Verhaltens nur zum Teil als authentischer Ausdruck subjektiver Motivationen und Präferenzen erklärt werden kann, im übrigen aber durch soziale Faktoren determiniert wird, deren erfolgreiche Pflege und Manipulation eine der wichtigsten Aufgaben der jeweiligen Versammlungs-

3 Diese abweisende «Wagenburgmentalität» ist am Abend des 29. Nov 2009 besonders drastisch ans Licht getreten, wo die Annahme der Minarettverbotsinitiative durch die Schweizer Stimmbürger die deutschen Zeitungsredaktionen zu äusserst heftigen einheitlich politisch-korrekten Ablehnungsreaktionen ("Schande über dieses Land») verleitet hat, während über $80 \%$ der Kommentare eine ebenso beherzte Zustimmung zum Schweizer Entscheid signalisierten.

4 Während die meisten Bürger im Umfragen das Netz als sehr wirkungsvolles Instrument für politische Artikulation, Diskussion, Kampagnenführung und Organisation betrachten, wird ihm für die vertikale Kommunikation und Einflussnahme (mit politischen Eliten oder der öffentlichen Verwaltung) bezeichnenderweise wenig Bedeutung beigemessen - offensichtlich weil hier negative Erfahrungen dominieren (vgl. Oblak 2003). 
und Tagungsleitung bildet. Im Gegensatz dazu werden Online-Teilnehmer vor ihrem Heim- oder Bürocomputer erheblich weniger durch derartige exogene Faktoren beeinflusst: weil beispielsweise kein Druck verspürt wird, durch äusserliche Konformität mit anderen Reaktionen des Tadels und der Missbilligung zu vermeiden (Geser 2001; 2002).

Jede Analyse der Internetnutzung muss bei dieser weltweit universellen Basissituation beginnen: ein Mensch sitzt alleine vor dem PC, ständig herausgefordert, selbständig wählen zu müssen, und absorbiert durch die interaktive Welt, die sich inm dort öffnet. Häufig genug wirkt das Internet depolitisierend in dem Sinne, dass der Nutzer mehr Möglichkeiten sieht, durch eigenes individuelles Handeln Ziele zu erreichen, für die bisher ein politisches Engagement nötig gewesen wäre: was soll ich mich für liberalere Copyrights einsetzen, wenn ich ohnehin die Möglichkeit habe, die neuesten Filme in File Sharing Netzwerken kostenlos herunterzuladen?. Gleichzeitig wird es den Nutzern aber auch erleichtert, sich unabhängiger von räumlichen und strukturellen Gegebenheiten an politischen Prozessen mitzubeteiligen : indem sie beispielsweise in Diskussionsgruppen mitdiskutieren, Petitionen unterschreiben, Protestschreiben versenden oder Mitgliederbeiträge entrichten, ohne ihren Bürostuhl oder Sitzplatz am häuslichen PC zu verlassen, ja ohne ihre übrigen Rollenaktivitäten wesentlich zu unterbrechen (Grossman 1995, 147). Als Folge davon erhält der politische .Bereich erhält Zufluss von momerntanen Motivationen, die früher subpolitisch geblieben sind. z. B. spontane Empörungsreaktionen über Verbrechen, Katastrophen usw, die sich in direkte politisch Forderungen transformieren.

Ebenso erleichtert das Internet neue Formen des kollektiven Handelns, die kein Sicheinfinden an öffentlichen Plätzen (oder halböffentlichen Lokalen) notwendig machen und deshalb auch nicht zum sinnlich greifbaren und medial wirksamen physischen Phänomen einer «Versammlung» (bzw. gar eines «Menschenauflaufs») führen (Geser 2002). Dadurch wird es einerseits möglich, einerseits zusätzliche, bisher unbeteiligte Bevölkerungssegmente zu aktivieren. Andererseits aber bleibt das verbindliche kollektive Engagement. das in der Politik gemeinhin gefordert wird, auf einem brüchigen Fundament, weil damit zu rechnen ist, dass unmittelbare situative Lebensumstände, momentane Stimmungen und aktuelle kognitive Orientierungen tiefgreifend das subjektive Denken und individuelle Handeln bestimmen, weil jeder Einzelne ohne soziale Mediatisierung direkt aus seiner eigenen Lebenswelt heraus (re)agiert.

Rein kognitiv besteht das Problem, den Umfang und die Zusammensetzung irgendeines Kollektivs, das sich nur Online zusammenfindet, adäquat zu überblicken, sowie die adäquate Rezeption einer ausgesendeten Botschaft zu verifizieren (Lebkwsky 1999). Und auf motivationaler Ebene besteht für Nguyen and Alexander $(1996,116)$ das Hauptpoblem in der unverbindlichen Beliebigkeit von Online-Inter- 
aktionen «[because they] lack the responsibility of an actual bodily commitment». So fehlt ihnen der dauernde Feedback missbilligender Blicke, die physisch anwesende Sprecher davon abhält, endlos oder in feindseliger Weise zu sprechen (Bregman und Koay 2000): mit der Folge, dass aus Mangel an horizontaler Sozialkontrolle potente Mittel zentralistischer Steuerung («Moderation») notwendig sind, um überbordende Postings oder rücksichtslose «Flamings» zu unterbinden. Dank der grossen Relevanz internal-subjektiver Faktoren hängt das virtuelle wahrscheinlich stärker als das realweltliche Kommunikationsverhalten von subjektiven Dispositionen und Fähigkeiten ab, die der pädagogischen Formung zugänglich sind: z.B. von der erfolgreichen Einsozialisierung einer "Netiquette», die auch ohne dauernde Beobachtung durch andere (d.h. auf der Basis reiner «Innenlenkung») hinreichend funktioniert, oder von der vorgängigen Schaffung von Lernbereitschaften, die in hohem Masse bestimmen, ob und in welchem Umfang man das Internetsurfen für den Wissenserwerb (anstatt z. B. bloss für unterhaltende Zwecke) nutzt (Delli Carpini und Scott 2002). Das Internet erhöht den Grad, in dem das Wissen und Können eines Menschen allein von seinen subjektiven Motivationen und Interessen abhängig ist: eben weil objektiv-physische, situative Bedingungen an Bedeutung verlieren. Wer politisch interessiert ist, wird das Netz produktiv zur Ausdehnung seines politischen Wissens nutzen und sich damit immer stärker von politisch Uninteressierten unterscheiden.

All diesen Tendenzen zur Individualisierung waren im frühen Web 1.0 noch dadurch Grenzen gesetzt, dass (z. B. in Form von «Home Pages» oder "Chat Rooms» etc.) noch Orte des virtuellen kollektiven Zusammenfindens und der Gemeinschaftsbindung geschaffen worden sind, die sich an der Metaphorik des physischen Raumes orientierten. Demgegenüber hat sich im Web 2.0 mit den «Social Network Sites» (z. B. Facebook) eine nicht mehr an virtuellen Orten, sondern an individuellen Personen orientierte Form der Vernetzung etabliert. Indem jeder Einzelne sich primär als Zentrum eines eigenen Freundschaftsnetzwerks definiert, wird eine fundamentalere, schwerer überwindbare Kluft zur Politik geschaffen, die ja immer eine Überschreitung solch informeller Gruppierungen (in Richtung auf verbindlichere formelle Interaktionen) impliziert.

Durch diese neuen Online-Angebote bekommen individuelle Politiker einen neuen Kanal, auf dem sie sich direkt auf Augenhöhe mit und nicht mehr im Windschatten ihrer Partei präsentieren können. Sie setzen ihre Agenda selbst und erreichen online viel leichter Aufmerksamkeit. Daraus ergeben sich neben Chancen auch Risiken. Wenn Hubertus Heil (SPD) und Cem Özdemir (Grüne) ihre virtuelle Stimme erheben, dann schenken ihnen mehrere tausend Menschen Aufmerksamkeit. Bislang konnten Heil auf Twitter und Özdemir auf Facebook deutlich mehr Follower und Unterstützer (virtuelle 
Kontakte) gewinnen, als es ihren Parteien auf den jeweiligen Plattformen möglich war. (Roggenkamp 2009).

Zweifellos profitieren Parteien insofern, als sie mittels solcher Einzelexponenten in viel aktiverer und flexiblerer Weise als mit Hilfe kollektiv erarbeiteter Verlautbarungen und «Resolutionen» an öffentlichen Diskursen teilnehmen können. Sie bezahlen dafür aber mit dem Risiko, dass keine konsistent-einheitliche Aussendarstellung mehr gelingt.

Generell hängt die faktische Nutzung der Online-Medien also wie die Wirkung der mittels ihrer getätigten Kommunikationen weitgehend von Faktoren ab, die nicht in den Computernetzen selber erzeugt werden können: z. B. von Effekten kollektiver Mobilisierung und Emotionalisierung, wie sie nur im Rahmen konventioneller Protestaktionen erreichbar sind.

The Net.does not replace the picket line, mass meeting, occupation, leaflet or collecting bucket. Try standing in a shopping center asking people to go home and type http://www.gn.apc.org/labournet/docks. (Dropkin 1996)

So können Vereinigungen mit hoch motivierter Anhängerschaft selbst bei minimaler Qualität ihres Webauftritts hohe Besucherzahlen vermelden, während Vereinigungen mit apathischer Mitgliederbasis auch bei beliebig hohem Geldaufwand für professionelle Webgestaltungen keinen Erfolg verzeichnen (Werber 1998). Daraus ergibt sich, dass die mit der neuen Netzkommunikation verknüpften Einflusschancen einseitig jenen (rein informell konstituierten) sekundären Eliten («geek adhocracies») zuwachsen, deren Mitglieder psychologisch überdurchschnittlich motiviert sind, sich dauerhaft in eine bestimmte Richtung zu engagieren (Klein 2000; Geser 2001, 40) und dadurch leicht in der Lage sind, sich auf Kosten der gewöhnlichen Mitgliederbasis beträchtlichen Einfluss zu verschaffen (Liff 1998). Wo immer ein Diskussionsforum eingerichtet wird, wird es von einer meist kleinen Oligarchie selbsterwählter Aktivisten in Beschlag genommen, so dass die Führungselite fälschlicherweise glaubt, in ihren Email-Stellungnahmen «des Volkes Stimme» zu vernehmen (Liff 1998; Klein 2000; Geser 2001, 40).

\section{Die unbeschränkte Gegenwärtigkeit vergangener Kommunikationen als Quelle der Strukturbildung und sozialen Kontrolle}

Eine bisher zu wenig beachtete, überaus folgenreiche Eigenschaft der neuen Netzöffentlichkeit besteht darin, dass alle in früheren Kommunikationsakten jemals erfolgten Äusserungen im Prinzip ungeachtet ihres Zeitpunktes auf derselben medialen Ebene präzis und ohne besonderen Aufwand beliebig lange zugänglich bleiben: so dass Altes von Neuem bloss überlagert wird, ohne von ihm in den 
Hintergrund gedrängt oder gar endgültig eliminiert zu werden. Damit steht sie im Kontrast zu den Massenmedien, wo vergangene Zeitungen und Sendungen sehr rasch deaktualisiert werden und in schwer zugänglichen Archiven verschwinden, wie auch zum mündlichen Diskurs, wo jeder Sprecher tendenziell nur auf unmittelbar vorangehende Voten reagiert. Indem sie ihren Nutzern Informationsbestände aus verschiedensten Zeiträumen in genau derselben Weise verfügbar machen, können Computernetze der politischen Öffentlichkeit eine bisher unbekannte zeitliche Tiefendimension verleihen, die beispielsweise für die kontinuierliche Akkumulation von Wissensbeständen, die Ausreifung von Argumentationsstrukturen, die Rückbeziehung auf frühere geschichtliche Perioden und die Inganghaltung einer eigenen Systemevolution genutzt werden kann. kann. Typischerweise verlangen Online-Diskurse (idealiter) eine gesteigerte rezeptive Aufmerksamkeit und Reaktionsbereitschaft, insofern (im Vergleich mit mündlichen Diskussionen) weniger voraussehbar ist, auf welche vorangegangenen Kommunikationen Votanten Bezug nehmen (werden), und die für orale Kommunikationsflüsse charakteristischen linearen Abläufe werden durch komplexere Vernetzungen ersetzt, indem Voten simultan auf mehrere bisherige Voten Bezug nehmen, sie durch Collage in eine Synopsis bringen oder durch Zusammenfassung auf eine neue Generalisierungsstufe heben.Im politischen Diskurs sind vor allem die Zuwächse an Sozialkontrolle bedeutsam, die beispielsweise dadurch entstehen, dass Votanten für alles, was sie früher gesagt haben, ad infinitum haftbar gemacht werden und damit die frühere Freiheit verlieren, straflos inkonsistent zu sein oder gar zu völlig neuartigen, gegensätzlichen Standpunkten überzuwechseln. Diese erbarmungslose Netzkontrolle wurde erstmals 1995 im «Presidential Memory Project» des amerikanischen «National Public telecomputing Network» realisiert, das den Zweck hatte, alle Dokumente über Präsident Clinton (seine Laufbahn, seine Wahlkampagne, seine Amtsführung usw.) zu aggregieren und während seiner gesamten Amtszeit abrufbar zu halten (Geser 1996). Die offensichtliche Absicht derartiger «monitoring systems» besteht darin, amtierende Politiker zu einer erhöhten «Selbsttreue» und zeitlichen Konsistenz ihrer Ansichten und Verhaltensweisen zu verpflichten bzw. sie im Falle von Abweichungen unter einen schärferen Begründungszwang zu setzen. Falls sich derartige Bewertungskriterien durchsetzen, könnten Politiker leicht an der Praktizierung anderer geschätzter Tugenden gehindert werden: etwa der Bereitschaft, sich ungeachtet der Vergangenheit immer wieder neu an aktuelle Problemlagen, politische Stimmungslagen im Lande etc. zu adaptieren.

Generell stehen solch konservierende Einflüsse in einem spannungsvollen Verhältnis zur wachsenden Differenzierung der Lebensphasen und zur Dynamisierung moderner Biographien, die zunehmend Raum fordern, um sich im Interesse eine innovativen Neubeginns vom Ballast persönlicher Vergangenheiten zu befreien. 
Wie die folgende Spiegel-Nachricht vom 8.8.2009 illustriert, fordert gerade der Eintritt in ein politisches Amt häufig die Zulegung einer gesteigert seriösen Neuidentität, die mit «kompromittierenden» Aspekten des früheren Privatlebens kollidiert:

Harald Christ, der SPD-Mittelstandsbeauftragte des Kanzlerkandidaten Frank-Walter Steinmeier, hat mehrere seiner Internetauftritte gelöscht. Nach seiner Nominierung ins Schattenkabinett ... hat der Unternehmer und Multimillionär aus Rheinland-Pfalz eine Webseite aufgegeben, auf der er für Events und Empfänge in seiner Villa in Südafrika warb... Auf der Seite wurde dem Bericht zufolge die Villa über den Hügeln von Kapstadt als Ort 〈für kulinarische Events〉, ‘formelle Empfänge〉 oder als Ferien-Domizil «mit Butler-Services angepriesen. ...Videos auf der Seite zeigten die exklusiven Räumlichkeiten, in denen afrikanische Diener Champagner umhertragen. Christ selbst sagte: «Seit meiner Berufung ins Steinmeier-Team habe ich lernen müssen, dass man als Unternehmer in Deutschland nicht nur Freunde hat. Deshalb habe ich die Belair-Constantia-Seite, wie alle meine privaten Aktivitäten, auch die in Netzwerken wie Xing oder Facebook, bis auf weiteres vom Netz genommen.)

Besondere Risiken entstehen, wenn sich Mandatsträger in eigenen Blogs freimütig zu vielerlei Tagesthemen äussern oder sich in Chats zu spontanen Äusserungen hinreissen lassen, die mit ihrer beruflichen Verpflichtung zur konsistenten Selbstdarstellung, Parteiloyalität und politischen Korrektheit im Spannungsverhältnis stehen. ${ }^{5}$ So mag gerade die grenzenlose Geschwätzigkeit des Netzes politische Amtsträger zu einem schweigsameren Habitus hindrängen, um Angriffsflächen auf ihre Person zu vermindern und ihnen die Freiheit zu lassen, allfällige Meinungsoder Verhaltensänderungen ohne ausführliche Rechtfertigungen zu vollziehen.

\section{Die Loskoppelung politischer Kommunikation vom politischen Handeln}

Aus vielerlei Gründen hat der niederschwellige und unverbindliche Zugang zur politischen Kommunikation zur Folge, dass der Aufbau handlungsfähiger kollektiver Aktivitätsformen und Organisationsstrukturen schwieriger wird, die für effektives politisches Handeln die Voraussetzung bilden,

Erstens wird die innere Kohäsion und Organisationsfähigkeit politischer Organisationen dadurch beschränkt, dass sie ihre Mitglieder ähnlich wie kommerzielle Firmen ihre Kunden keiner bedeutsamen Sozialkontrolle und autoritativen Füh-

5 So musste der Präsident der «Schweiz Volkspartei» in der Stadt Luzern 2009 von allen Ämtern zurücktreten, nur weil er sich auf seiner Homepage abschätzig über die mangelnde Gepflegtheit einheimischer Frauen geäussert hatte (NZZ Online 12. August 2009; 08.50) 
rung unterwerfen können, weil diese jederzeit in der Lage sind, auf Meinungsverschiedenheiten und Unzufriedenheiten mit Inaktivität und Abwanderung (d. h. mit «exit» anstatt «voice») zu reagieren. Dementsprechend bleibt ihre Aktionsfähigkeit zu jedem Zeitpunkt an einen freiwilligen Konsens gebunden. Selbst millionenfach «unterzeichnete» Online-Petitionen entfalten keine Wirkung, weil man davon ausgehen, dass sich die teilnehmenden Stubensurfer von spontan-volatilen Momentanreaktionen leiten liessen und kein Commitment signalisieren, das z. B. mit der Teilnahme an einer Demonstration vergleichbar wäre

Zweitens fehlt den (ausschliesslich) digitalen politischen Akteuren das kollektive Konfliktpotential, das bei realweltlichen Organisationen aus ihren Einflussmöglichkeiten auf die physische Umwelt entsteht. Streiks, Demonstrationen, Sit ins und andere Störaktionen müssen durch viel weichere Aktionen (z. B. Petitionen) ersetzt werden, die von den politischen Adressaten leicht ignoriert werden - auch wenn sie mit hoher Reichweite und zeitlicher Unmittelbarkeit wettzumachen versuchen, was ihnen an physischer Durchschlagskraft und öffentlicher Auffälligkeit fehlt. Die von «Weblogestan» ausgehende Soft Power im asymmetrischen Krieg mit der «Hard power» territorial basierter politischen Autoritären höchstens temporäre taktische Gewinne zu verbuchen.

Drittens erweisen sich webgestützte politische Aktionen zumindest deshalb als kurzlebig und unwirksam, weil über die hinter einer Webplattform stehenden Akteure und Gruppierungen (bezüglich Mitgliederzahl, Beziehungsnetzwerken Ressourcen etc.) häufig keine Klarheit besteht, und die meisten von ihnen von unbezahlten Freiwilligen unterhalten werden, deren Engagement sich auf nebenberufliche Randstunden, kurzfristige Zeiträume und partikuläre Zielsetzungen beschränkt. (Parker und Song 2006, 192). Die Tatsache, dass die Anfangsphasen politischer Online-(?)Mobilisierung ohne Aufbau formaler Organisation und beruflicher Rollenstrukturen vonstatten gehen können, erweist sich in den späteren Phasen als grosser Nachteil, wo eine derartige Organisation erforderlich wäre, um politische Handlungsstrategien auf Dauer zu stellen und im institutionellen Gefüge der Gesellschaft eine verlässliche Rolle (z. B. durch Einsitznahme in wichtige Gremien) zu übernehmen. Mangels gemeinsamer Führung, Zielsetzung, Regeln und organisatorischer Rahmenstrukturen bleiben rein digitale Bewegungskollektive nach aussen hin unscharf begrenzte und nach innen polymorph zusammengesetzte Aggregate, wo sich autonom bleibende Subgruppen in wechselnden Koalitionen zu kurzfristigen Ad-hoc-Aktionen zusammenfinden, ohne sich unter umfassendere und längerfristige Ideologen und Programme zu subordinieren. Dementsprechend verbleiben sie in einem «subinstitutionellen» Zustand, der es ihnen oft verunmöglicht, den von ihnen attackierten Akteuren überhaupt klare Forderungen 
zu stellen, auf sie in kontinuierlich-systematischer Weise Druck auszuüben oder gar mit ihnen in geregelte Verhandlungen zu treten. ${ }^{6}$ So ist die in der Schweiz 1997 gegründete «Internetpartei» bereits 2000 aufgelöst werden, weil es ihr in ihrer Beschränkung auf Onlinekommunikation nicht gelungen ist, hinreichende Mobilisierungs- und Integrationskräfte zu erzeugen. Ganz im Gegensatz dazu steht z. B. die aktuelle Deutsche Piratenpartei, die ihre Websites zum grossen Teil dafür einsetzt, um auf breiter Front lokale Standaktionen, Versammlungen, Begegnungen und Demonstrationen zu organisieren.?

Aus den stürmischen Ereignissen um Thilo Sarrazin (vom Spätsommer 2010) kann man schliessen, dass wahrhaft grosse öffentliche Kampagnen immer noch bevorzugt durch traditionelle Medienerzeugnisse (z. B. Sachbücher) ausgelöst werden, dass sie aber dank der Online-Kommunikation eine viel breitere und vielschichtigere Resonanz auslösen. So konnte sich neben dem einheitlichen Verdammungsurteil seitens der politisch korrekten Politiker-, Parteien- und Medienszene auch die dazu teilweise völlig konträren Meinungen in der Bevölkerung hinreichend Gehör und Sichtbarkeit verschaffen, und die Parteieliten sahen sich unvermittelt mit einer sich via Email lautstark rebellisch artikulierenden Mitgliederbasis konfrontiert.

Andererseits trägt genau diese Vielfalt der Online-Stimmen dazu bei, dass sie politisch wenig wirksam bleiben, denn so sehr das Internet hilfreich ist, um der ganzen Vielfalt gesellschaftlicher Denkweisen, Meinungen, Bewertungen Interessen oder Forderungen Ausdruck zu verleihen, so wenig kann es dazu beitragen, diese Vielfalt zu jener einheitlichen «öffentlichen Meinung» zu aggregieren, die nach Ansicht des Philosophen John Locke die höchste Machtinstanz im Staate darstellen soll. Vielmehr wird der für entschlossenes politisches Handeln nötige kollektive Konsens schwerer erreichbar, weil vielfältigere Minderheitsmeinungen berücksichtigt werden müssen (Lebkowsky 1999); und die Stellung von Partei- und Verbandeliten wird geschwächt, weil sie über keinen privilegierten Zugang zur Öffentlichkeit (via «Zentralorgan») mehr verfügen (Geser 2001). So könnte die Netzkommunikation paradoxerweise den Zentralisierungsgrad politischer Macht durchaus verstärken: weil Politiker für beliebige Entscheidungspositionen irgendwo im Netz eine plebiszitäre Unterstützungsbasis finden, weil sie sich auf ihre eigene Meinung zurückverwiesen sehen, da sie aus der Kakophonie der Netzstimmen keine klare «Volksmeinung» heraushören können, oder einfach: weil ihre Autorität gefragt ist, um

6 Falls überhaupt kollektive Aktionen erfolgen, haben sie meist den expressiven Charakter spontaner "flash campaigns», die. im Gegensatz zu instrumentalen Aktionen nicht auf die Erreichung bestimmter Ziels, sondern nur der öffentlichen Kundgabe von Gesinnungen und Meinungen ausgerichtet sind. Diese Beschränkung hat den zweifachen Vorteil, dass keine Diskussionen über Zielsetzungen, Strategie und Taktik erforderlich sind, und dass sich alle Teilnehmer unabhängig ihrer unterschiedlichen Fähigkeiten in gleicher Weise beteiligen können

7 http://www.piratenpartei.de/ 
endlose unfruchtbare Online-Diskurse durch ein Machtwort zu beenden (Bregman und Koay 2000).

Ungeachtet ihrer fraglichen politischen Effektivität können die politischen OnlineDiskurse in medienpädagogischer Sichtweise als sehr niederschwellig zugängliche Übungsfelder betrachtet werden, in der sich unzählige bisher politisch völlig inaktive Personen lernen können, sich reflexiv ihrer eigenen politischen Standpunkte zu vergewissern, sie in intersubjektiv verständliche sprachliche Form zu kleiden und unter Beachtung zivilgesellschaftlicher Regeln des Respekts und diskursiven Rationalität auf die Äusserungen Anderer zu reagieren (Dahlberg 2001). Allerdings ist ihrer sozialisatorische Kraft keinesfalls mit derjenigen primärer Gruppenkommunikation zu vergleichen, weil in den verbalen Austauschprozessen zu wenig interpersonelle Sozialkontrolle erfolgt und jederzeit leicht zugängliche Exit-Optionen verfügbar sind. In pädagogischer Perspektive wird es deshalb umso wichtiger, dass den Netzteilnehmer ex ante ein hinreichendes Kompetenz- und Sozialisationsniveau zu vermitteln.

\section{Literatur}

Arendt, Hannah. 1968. Between Past and Future: Eight exercises in political thought. New York: Viking.

Bimber, Bruce Allen und Richard Davis. 2003. Campaigning online: The Internet in U.S. elections. New York: Oxford University Press.

Brundidge, Jennifer. 2008 «The Contribution of the Internet to the Heterogeneity of Political Discussion Networks: Does the Medium Matter?» Paper presented at the International Communication Association, Dresden, Germany: http:// www.allacademic.com//meta/p_mla_apa_research_citation/2/3/2/1/0/pages232107/p232107-1.php (31.5.2012).

Dahlberg, Lincoln. 2001. "Computer-mediated Communication and The Public Sphere: a Critical Analysis.» Journal of Computer Mediated Communication 7 (1): http://jcmc.indiana.edu/vol7/issue1/dahlberg.html (31.5.2012).

Delli Carpini, Michael X. und Scott Keeter. 2002. "The Internet and an Informed Citizenry.» In The Civic Web, edited by David Anderson and Michael Cornfield, 129-153. Lanham, MD: Rowman und Littlefield.

De Sola Pool, Ithiel. 1998. Politics in Wired Nations. New Brunswick: Transaction Publisher.

Donath, Judith S. 1999. "Identity and Deception in the Virtual Community.» In Communities in Cyberspace, edited by Marc A. Smith und Petert Kollock, 2959. London: Routledge.

Fraser, Nancy. 1991. "Rethinking the Public Sphere: A Contribution to the Critique of Actually Existing Democracy.» In Habermas and the Public Sphere, edited by Craig Calhoun, 109-42. Cambridge, MA: MIT Press. 
Grossman, K. Lawrence. 1995. The Electronic Republic: Reshaping democracy in the information age. New York: Viking.

Geser, Hans. 1996. "Auf dem Weg zur «Cyberdemocracy»: Auswirkungen der Computernetze auf die öffentliche politische Kommunikation.» Sociology in Switzerland: Towards Cybersociety and Vireal Social Relations. Online Publications. Zürich: http://socio.ch/intcom/t_hgeser00.htm (31.5.2012).

Geser, Hans. 2001. "On the Functions and Consequences of the Internet for Social Movements and Voluntary Associations.» Sociology in Switzerland: Social Movements, Pressure Groups and Political Parties. Online Publications. Zürich (Release 2.0): http://socio.ch/movpar/t_hgeser3.pdf (31.5.2012).

Geser Hans. 2002. «Towards a (Meta-)Sociology of the Digital Sphere.» Sociology in Switzerland: Towards Cybersociety and Vireal Social Relations. Online Publications. Zürich: http://socio.ch/intcom/t_hgeser13.htm (31.5.2012).

Habermas, Jürgen. 1962. Strukturwandel der Öffentlichkeit. Untersuchungen zu einer bürgerlichen Kategorie der Gesellschaft. Neuwied: Luchterhand.

Hall, Kira. 1996. "Cyberfeminism». In Computer-mediated Communication: Linguistic, social and cross-cultural perspectives, edited by Susan C. Herring, 147170. Amsterdam: John Benjamins.

Hill, Kevin A. und John E. Hughes. 1998. Cyberpolitics: Citizen activism in the age of the Internet. Lanham: Rowman und Littlefield.

Jordan, Tim. 1999. Cyberpower: The culture and politics of cyberspace and the Internet. London: Routledge.

Katz, Jon. 1997. «Birth of a Digital Nation.» Wired 5 (4): 49-52; 184-191.

Klein, Naomi. 2000. "Were the DC and Seattle Protests Unfocused or Are Critics Missing the Point? The Vision Thing.» The Nation, 10 July, 18-21.

Lebkowsky Jon. 1999. "A Few Points about Online Activism.» Cybersociology Magazine 5: http://www.cybersociology.com/files/5_JonLebkowsky.html (31.5.2012).

Lea, Martin und Russell Spears. 1991. "Computer-mediated Communication, deindividuation and group decision-making.» International Journal of Man-Machine Studies 34 (2): 283-301.

Liff, Allen. 1998. "Fostering Online Collaboration and Community.» Association Management 50 (9): 33-39.

Mill, John Stuart. 1859, 1998. On Liberty and Other Essays. Oxford: Oxford University Press.

Min, Seong-Jae. 2007. "Online vs. Face-to-Face Deliberation: Effects on civic engagement.» Journal of Computer-mediated Communication 12 (4): http:// jcmc.indiana.edu/vol12/issue4/min.html (31.5.2012).

Mutz, Diana C. und Paul S. Martin. 2001. «Facilitating Communication Across Lines of Political Difference.» American Political Science Review 95 (1): 97-114. 
Nguyen, Dan Thu und Jon Alexander. 1996. "The Coming of Cyberspacetime and the End of the Polity.» In Cultures of Internet: Virtual spaces, real histories, living bodies, edited by Rob Shields, 99-124. London: Sage.

Nielsen Wire. 2010. What Americans Do Online: Social Media And Games Dominate Activity. August $2^{\text {nd }}$. http://blog.nielsen.com/nielsenwire/online_mobile/what-americans-do-online-social-media-and-games-dominate-activity/ (31.5.2012).

Oblak, Tanja. 2003. «Boundaries of Interactive Public Engagement: Political Institutions and Citizens in New Political Platforms.» Journal or Computer-mediated Communication 8 (3): http://jcmc.indiana.edu/vol8/issue3/oblak.html (31.5.2012).

Postmes, Tom, Spears, Russell and Lea, Martin. 1998. «Breaching or building social boundaries? SIDE-effects of computer-mediated communication.» Communication Research 25 (4): 689-715.

Price, Vincent / Cappella, Joseph N. 2002. "Online Deliberation and Its Influence: The electronic dialogue project in campaign 2000.» IT und Society 1 (1): 303329.

O'Brien, Jodi. 1999. «Writing in the Body: Gender (re)production in online interaction.» Communities in Cyberspace, edited by Marc A. Smith und Peter Kollock, 76-104. London: Routledge.

Rice, Ronald E. 1993. "Media Appropriateness: Using social presence theory to compare traditional and new organizational media.» Human Communication Research 19 (3): 451-484.

Robins, Kevin. 1996. "Cyberspace and the World We Live In.» In Cyberspace, Cyberbodies and Cyberpunk: Cultures of technological embodiment, edited by Mike Featherstone und Roger Burrows, 135-155. London: Sage.

Roggenkamp, Klaus W. 2009. «Die Vielstimmigkeit der Parteien im Internet.» Welt Online, 25. Mai 2009.

Spears, Russel und Lea, Martin. 1992. "Social Influence and the Influence of the 〈Social» in Computer-mediated Communication.» In Contexts of Computermediated Communication, edited by Martin Lea, 30-65. London: HarvesterWheatsheaf.

Sunstein, Cass R. 2001. Republic.com. Princeton, NJ: Princeton University Press.

Streck, John M. 1998. «Pulling the Plug on Electronic Town Meetings: Participatory democracy and the reality of the usenet.» In The Politics of Cyberspace: A new political science reader, edited by Chris Toulouse and Timothy W. Luke, 18-47. New York: Routledge. 